\title{
Cross-language parafoveal semantic processing: Evidence from Korean-Chinese bilinguals
}

\author{
Aiping Wang ${ }^{1,2} \cdot$ Junmo Yeon ${ }^{1} \cdot$ Wei Zhou ${ }^{3} \cdot$ Hua Shu ${ }^{4} \cdot{\text { Ming } \text { Yan }^{5}}^{5}$
}

Published online: 30 June 2015

(C) Psychonomic Society, Inc. 2015

\begin{abstract}
In the present study, we aimed at testing crosslanguage cognate and semantic preview effects. We tested how native Korean readers who learned Chinese as a second language make use of the parafoveal information during the reading of Chinese sentences. There were 3 types of Korean preview words: cognate translations of the Chinese target words, semantically related noncognate words, and unrelated words. Together with a highly significant cognate preview effect, more critically, we also observed reliable facilitation in processing of the target word from the semantically related previews in all fixation measures. Results from the present study provide first evidence for semantic processing from parafoveally presented Korean words and for cross-language parafoveal semantic processing.
\end{abstract}

Keywords Parafoveal $\cdot$ Semantics $\cdot$ Korean $\cdot$ Chinese Bilingual

Reading is a complex task that involves movement of the eyes to different locations across the text for lexical processing.

Ming Yan

mingyan@uni-potsdam.de

1 School of Psychology, Beijing Normal University, Beijing, People's Republic of China

2 Beijing Key Laboratory of Applied Experimental Psychology, Beijing Normal University, Beijing, People's Republic of China

3 Department of Psychology, College of Education, Capital Normal University, Beijing, People's Republic of China

4 State Key Laboratory of Cognitive Neuroscience and Learning, Beijing Normal University, Beijing, People's Republic of China

5 Department of Psychology, University of Potsdam, Karl-Liebknecht-Str. 24/25, 14476 Potsdam-Golm, Germany
Low-level orthographic and high-level semantic information from the currently fixated word, as well as words in the parafovea that are not yet recognized, are activated during fixations. The gaze-contingent boundary paradigm (Rayner, 1975) has been widely used to investigate parafoveal processing. In this paradigm, parafoveal preview of a target word is either provided in its original form or masked by an alternative word (or a nonword) in the position of the target word. The preview is replaced by the correct target word immediately after readers' eyes cross an invisible boundary located between the pretarget and target words. Preview benefit (PB) is indicated by shorter fixation durations on the target word when the preview is provided compared to when it is masked. PB has been documented for various types of information overlap between the preview and the target, such as orthography and phonology (Schotter, Angele, \& Rayner, 2012). The present study demonstrates activation of cross-language parafoveal semantics for Korean-Chinese bilinguals during reading.

Traditionally, evidence for parafoveal processing of semantics has been elusive in English in word-pair reading (e.g., Inhoff, 1982; Inhoff \& Rayner, 1980) and in sentence reading tasks (Rayner, Balota, \& Pollatsek, 1986; but Schotter, 2013). Yan, Richter, Shu, and Kliegl (2009) proposed that the mapping between orthography and semantics in a writing system plays an important role in parafoveal processing: the absence of semantic PBs in English could be because semantic information is normally available relatively late (i.e., via phonological mediation) in lexical processing and thus the absence of evidence for semantic preprocessing may not be language universal. As a logographic writing system, Chinese orthography generally maps more closely to meaning than to sound (Hoosain, 1991) and there is evidence for direct access to semantics bypassing phonological activation in Chinese (e.g., Chen \& Shu, 2001; Zhou \& Marslen-Wilson, 2000). 
Indeed, Yan et al. (2009) demonstrated semantic PB using simple Chinese characters as previews. Later studies extended this effect to other character sets (Tsai, Kliegl, \& Yan, 2012; Yan, Zhou, Shu, \& Kliegl, 2012; Yang, Wang, Tong, \& Rayner, 2012).

The studies reviewed above all focused on parafoveal processing of readers' native language. The present study goes beyond this level and tests whether high-level parafoveal semantic information from one writing system can be obtained to facilitate processing of the target word in a different writing system among bilingual readers. ${ }^{1}$ We consider this as a more powerful test for parafoveal high-level information processing because cross-language semantic activation is obviously a more demanding task. Previously, Altarriba, Kambe, Pollatsek, and Rayner (2001) creatively employed noncognate translations and cognate translations as parafoveal previews and found no preview effect for the noncognate previews. There was, however, a preview effect for the cognates, but it was not significantly larger than that for orthographic controls. The authors concluded that the cognate PB was merely due to orthographic/phonological overlap between preview and target. Although the authors concluded that "the system discards semantic information when the preview is orthographically dissimilar" (Altarriba et al., 2001, p. 886), given the reliable evidence for parafoveal semantic processing in Chinese, we consider it likely that the failure to observe reliable crosslanguage semantic preview effect could be due two alternative reasons: (a) the alphabetic nature of Spanish and English scripts and/or (b) parafoveal preview being eccentric.

In the present study, we address the question of crosslanguage cognate and semantic preview effects by using Korean preview words during the reading of Chinese sentences. The Korean writing system offers a unique opportunity for testing these effects due to its relationship to the Chinese writing system. Hangul is the native alphabet and official script of the Korean language, and it was invented and promulgated in the 15th century during the reign of King Sejong. The Hangul is similar to the Chinese and Japanese but different from English with respect to spatial layout: Each Korean character occupies the same horizontal extent in the text, irrespective of visually complexity. However, different from Chinese, the Hangul is fundamentally alphabetic: Hangul characters are composed of letters that represent consonants and vowels but not meanings. Therefore, the Korean writing system is sometimes considered as a hybrid between European and Chinese writing systems. More interestingly, before the invention of Hangul, the Chinese writing system had been used for centuries. Most Korean scholars continued to write in Hanja (Chinese) when possible and to use Hangul mainly for grammatical suffixes and native

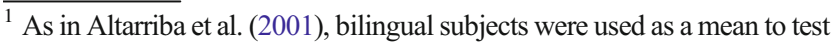
parafoveal semantic processing rather than to study bilingualism per se.
}

Korean words, even after the invention of Hangul, similar to Kanji and Kana in Japanese. As a consequence, there is a large set of words in the Korean vocabulary that originates from or is influenced by Chinese, making up over $60 \%$ of the Korean written vocabulary. Using pure Hangul to write became common in the 20th century: since 1970, the Korean government has been encouraging citizens to stop using Hanja and replace them with Hangul (Sohn, 2001). As a consequence, there is a set of cognate vocabularies in Chinese and Korean sharing a common origin of Chinese words, offering a unique opportunity to study cognate words without orthographic overlap.

The only study on parafoveal processing during Korean reading was conducted by $\mathrm{Kim}$, Radach, and Vorstius (2012): the previews had either correct or incorrect case markers in terms of syntactic category. Results showed parafoveally presented inappropriate syntactic case markers led to a substantial prolongation in processing time of the target word and thus provided the first evidence for parafoveal processing of syntactic information using the Korean script. Results from Kim et al. (2012) also suggest a possibility in preprocessing of other types of high-level linguistic information.

In the present study, we predict positive evidence for crosslanguage semantic preview effects among Korean-Chinese bilinguals. This is because, as reviewed above, information is more densely packed in Chinese/Korean as compared to alphabetic scripts. As a result, upcoming words in the parafovea are on average less eccentric in Chinese/Korean, allowing readers to benefit from the higher visual acuity in the near parafovea, which facilitates parafoveal processing. The cognate previews used in the present study are phonetic transcriptions of Chinese-originated words written in Hangul; therefore, the preview word (in Hangul) and the target word (in Chinese) refer to an identical concept, and they have very similar pronunciations in the two languages. Therefore, we predict that they should lead to a strong PB. However, because there is overlap between the preview and target words at both semantic and phonological levels, the nature of cognate $\mathrm{PB}$ is mixed. Therefore, we also designed a semantically related noncognate preview condition, for a test of pure parafoveal semantic activation, without any orthographic or phonological support.

\section{Method}

\section{Subjects}

Forty-seven subjects participated in the eye-tracking experiment. The subjects were on average 21 years old $(S D=2.1)$ and had studied Chinese for 3.9 years $(S D=2.3)$. Three independent samples of 10,15 , and 18 subjects participated in three norming studies for (a) semantic relatedness between 
the preview and target words, (b) predictability of the target words from sentence contexts, and (c) familiarity of the preview words. All subjects were native Korean readers with normal or corrected-to-normal vision and were undergraduate students from the Beijing Normal University who passed the Chinese Proficiency Test (also known as HSK-Hanyu Shuiping Kaoshi) Grade 4, which is the official standardized test of Chinese language level for nonnative speakers in the People's Republic of China.

\section{Material}

Thirty two-character Chinese words were chosen as the target words. For each target, three types of Korean words were selected serving as a cognate translation preview, which have an identical lexical representation as well as a very similar pronunciation to the target word but without any orthographic overlap; a semantically but not phonologically related preview; and finally, an unrelated preview. As shown in Table 1, a number of factors were controlled among the three conditions. The preview words were matched with respects to visual complexity as indexed by character stroke frequency (Zhang, Zhang, Xue, Liu, \& Yu, 2007), $F(2,89)=1.093, p>.1$, and by number of pixels, $(F<1)$, word frequency, $F(2,89)=1.295, p>.1$, and familiarity, $F(2$, $89)=1.084, p>.1$. Finally, as expected, the three types of preview words differed in their semantic relatedness to the target, $F(2,58)=819.227, p<.001$. Paired $t$ tests indicated a hierarchical pattern with greater semantic relatedness to the target words for the cognate previews than that for the related previews $(t=10.942, p<.001)$, which in turn was greater than that for the unrelated previews $(t=23.220, p<.001)$.

Table 1 Word properties

\begin{tabular}{lllll}
\hline & Target & Cognate & Related & Unrelated \\
\hline Word & 基本 & 기본 & 초급 & 무료 \\
Translation & basic & basic & beginning & free \\
Pronunciation & jiben & gibon & chogeub & mulyo \\
Word frequency & & $45(35)$ & $34(26)$ & $45(30)$ \\
Spatial frequency & & $5.4(0.7)$ & $5.4(0.7)$ & $5.2(0.7)$ \\
N. pixels & & $345(54)$ & $346(57)$ & $329(57)$ \\
Sem. rating & & $4.9(0.2)$ & $3.6(0.6)$ & $1.1(0.1)$ \\
Fam. rating & & $4.7(0.3)$ & $4.6(0.4)$ & $4.7(0.2)$ \\
\hline
\end{tabular}

Note. Means (and standard deviations, in parentheses) of word frequency (National Institute of Korean Language 2005), visual complexity (character stroke spatial frequency and number of pixels), semantic relatedness between the preview and target words, and familiarity of the preview words. Pronunciation of the preview words are given in Romaja, a system for representing the Korean language using the Latin script, and that of the target word is given in Pinyin, a phonetic system for transcribing Chinese characters into the Latin alphabet.
Three different sentence frames were constructed for each target word so that there were totally 90 experimental sentences. Using the target words for multiple times was mainly due to the difficulty in creating word pairs meeting the above criteria. Given that the sentences were 14 to 19 characters in length $(M=16.1, S D=1.3)$, it is unlikely for the subjects to notice the repetition of the targets. The pretarget words were also always two-character words. The pretarget and target words never appeared in the first three or the last three characters. Each sentence was presented only once to a subject, and the conditions were counterbalanced across subjects. Figure 1 shows a set of example sentences. The sentence contexts were designed to be nonpredictive for the targets, and this was confirmed by a cloze test in which the subjects were given sentence frames prior to the target words and asked to complete the sentence. The targets were only guessed $1.3 \%$ times.

\section{Apparatus}

Eye movements were recorded using an EyeLink Desktop system with a $35 \mathrm{~mm}$ lens running at $1000 \mathrm{~Hz}$. Sentences were presented in single lines on the vertical position one third from the top of the screen of a 21-inch Dell P1130 monitor (resolution, $1024 \times 768$ pixel; frame rate, $120 \mathrm{~Hz}$ ). Given these parameters, the display change should complete within $12 \mathrm{~ms}$ after the eyes crossed the invisible boundary. Subjects were seated comfortably with a chin rest and a forehead rest at a distance of $65 \mathrm{~cm}$ from the monitor. Each character occupied a $40 \times 40$ pixel grid and subtended 1.4 degrees of visual angle. All recordings and calibrations were done monocularly, based on the right eye, and viewing was binocular.

熟练掌握数学기본公式对于考试很重要。

Cognate Preview

熟练掌握数学초급公式对于考试很重要。_Related Preview

熟练掌握数学古豆公式对于考试很重要。

Unrelated Preview

熟练掌握数学基本公式对于考试很重要。 Target

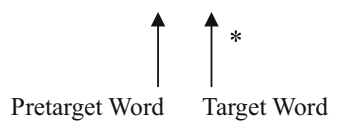

Fig. 1 A set of example sentences in the experiment. The preview words that are initially displayed in the target location are replaced by the target words immediately after the subjects' eyes (as indexed by the asterisks in the figure) crossed the invisible boundary, which is located between pretarget and target words. The target sentence translates as: Proficiency in the basic formulas in mathematics is very important for the exam 


\section{Procedure}

Subjects' gaze positions were calibrated with a standard 9point grid. Prior to the presentation of each sentence, the eye tracker checked for drift. If the eye tracker identified subjects' eyes fixating at the fixation point, the next sentence was presented with its first character occupying the position of the fixation point; otherwise, a recalibration was initiated. Participants were instructed to read each sentence for comprehension, then fixate on a dot in the lower right corner of the monitor and press a button to signal completion of the trial. As shown in Fig. 1, before the subjects' eyes crossed the invisible boundary located between the pretarget and target words, they got one of the three previews. The preview word was replaced by the target word immediately after the eyes crossed this boundary. Thirty randomly selected sentences were followed by an easy yes-no comprehension question, and on average subjects correctly answered $81 \%$ of them $(S D=8 \%)$.

\section{Data analysis}

Fixations were determined using an algorithm for binocular saccade detection (Engbert \& Kliegl, 2003). There were four levels of data screening: First, a total of 28 (i.e., $1 \%$ ) trials were removed due to subjects' blinking, coughing, or body movement during reading or tracker errors. Second, firstfixation durations (FFDs; durations of the first-fixations on words irrespective of number of fixations) shorter than $60 \mathrm{~ms}$ or longer than $800 \mathrm{~ms}$ and gaze durations (GDs; accumulative durations of fixations during the first-pass reading) longer than 1,200 ms were removed, excluding $3 \%$ of data in the target region. Third, trials with regressions from either pretarget or target words were discarded ( $8 \%$ ); this is because they may reflect incomplete parafoveal processing of preview words during fixations on pretarget words or incomplete foveal processing of target words. Finally, trials with late display change (i.e., in which the saccade crossed the boundary during the final $20 \%$ of the saccade duration) were excluded, because readers should be more likely to perceive a display change or a flash at this time (23\% trials).

Effect-size estimates are based on linear mixed models (LMMs) for durations and a generalized linear mixed model (GLMM) for skipping rate. Inferential statistics are based on planned comparisons for the cognate and related previews with the unrelated preview as a reference condition. We estimated model parameters of variance components for subjects and for items (sentences and target words) using the lmer program of the lme 4 package (version 1.1-7; Bates, Maechler, Bolker, \& Walker, 2014) in the R environment for statistical computing and graphics (version 3.1.1; R Development Core Team, 2014). In LMMs, estimates 1.96 times larger than their standard errors are interpreted as significant at the $5 \%$ level. This is because given the number of subjects and the large number of observations for each subject, the $t$ statistic in LMMs (i.e., M/SE) effectively corresponds to the $z$ statistic. We report log-transformed dependent variables of fixation times in the models because analyses of residuals and inspection of duration distributions strongly suggested that log-transformation is required to meet LMM assumptions (Kliegl, Masson, \& Richter, 2010). Analyses for untransformed and log-transformed durations yielded the same pattern of significance.

\section{Results}

Participants were more likely to skip the target words in the cognate preview condition $(b=.837, S E=.288, z=2.91$, $p=.003)$, and there was no difference between the related and unrelated previews $(b=.231, S E=.318, z=0.73$, $p=.467)$. A total of 2,913 observations contributed to the following duration analyses. Together with FFD and GD, we also included total reading time (TRT; sum of all fixation durations on the target word). Because very often GD includes refixations and TRT includes regressive rereading time, they are normally considered as relatively late processing measures (Inhoff, 1984), and thus these three fixation measures jointly provide an opportunity to test the time course of parafoveal information activation. As shown in Table 2, there were highly significant cognate PBs $(b=-.087, S E=.014, t=-6.28$; $b=-.193, S E=.016, t=-11.99$, and $b=-.216, S E=.016$, $t=-13.79$, for FFD, GD, and TRT, respectively).

A more critical test for cross-language parafoveal semantic activation is whether facilitation effects can be observed purely due to semantic relatedness between the preview and target words without phonological overlap. This is achieved by comparing the difference in processing time of the target word between the semantically related and the unrelated preview conditions. As expected and shown in Table 2, PBs from the related previews were also reliable across different duration measures $(b=-.030, S E=.014, t=-2.12 ; b=-.062, S E=.016$, $t=-3.81$, and $b=-.060, S E=.016, t=-3.77$, for FFD, GD, and TRT, respectively).

Table 2 Fixation measures

\begin{tabular}{llll}
\hline & \multicolumn{2}{l}{ Type of preview } & \\
\cline { 2 - 4 } & Cognate & Related & Unrelated \\
\hline Skip & $3.0(5.1)$ & $1.7(3.5)$ & $1.4(2.8)$ \\
FFD & $332(49)$ & $356(56)$ & $370(69)$ \\
GD & $451(83)$ & $517(106)$ & $554(119)$ \\
TRT & $454(84)$ & $535(117)$ & $572(130)$ \\
\hline
\end{tabular}

Note. Means (and standard deviations in parentheses) of first-fixation duration (FFD), gaze duration (GD), and total reading time (TRT). Values are computed across subjects' means. 


\section{Discussion}

The present study aimed at testing cross-language cognate and semantic preview effects. We observed a highly significant cognate $\mathrm{PB}$, replicating earlier findings of cognate facilitation (Altarriba et al., 2001; Libben \& Titone, 2009). Arguably, this is because words that are translations of each other are known to share a lexical representation in the two languages (e.g., Altarriba \& Mathis, 1997). Altarriba et al. (2001) found that the cognate preview did not lead to larger PB than the orthographic preview; the nature of their cognate preview effect was likely orthographic overlap. In the present study, although there was no orthographic overlap between the preview and the target words, the cognate preview effect may not be solely attributed to semantic preprocessing because there was also phonological overlap. Therefore, a more critical test for crosslanguage semantic preview effect was the semantically related noncognate preview condition. As expected, there were reliable PBs purely due to semantic overlap, which appeared from early on and lasted until second-pass reading. As compared to the Spanish and English preview words used in Altarriba et al. (2001), the Korean previews in the present study are presumably on average closer to the fovea, leading to a higher parafoveal processing efficiency. In addition, the present study may also benefit from a within-item design (i.e., cognate and noncognate translations are paired with the same target word), allowing for a better experimental control. Taken together, results from the present study suggest cross-language parafoveal semantic processing can be achieved independently of orthographic or phonological similarity.

The present result of semantic activation from parafoveally presented Korean words extends our knowledge on parafoveal high-level information processing. There is considerable additional evidence for parafoveal (morpho-)semantic processing in Chinese (Yan, Pan, Bélanger, \& Shu, 2015; Yan \& Sommer, 2015; Yen, Tsai, Tzeng, \& Hung, 2008). In contrast, such effects appear to be limited to synonym previews in English (Schotter, 2013) or to scripts with relatively transparent letter-phoneme correspondence, such as German (Hohenstein, Laubrock, \& Kliegl, 2010; Hohenstein \& Kliegl, 2014). Because alphabetic writing systems vary in their orthographic depth (i.e., the degree to which they are regular in their representation of sound), Hohenstein et al. (2010) argued that, as compared to English, a more transparent orthography-to-phonology mapping in German leads to faster phonological decoding, which in turn facilitates access to semantics during the short fixation periods. Korean is similar to German with respect to letter-to-phoneme correspondence: Both German and Korean are considered of intermediate orthographic depth. We adopt this argument to explain the semantic preview effect in Korean in the present study. It is important to notice that although semantic preview effects in German and Korean are superficially similar to those in Chinese, they are explained under fundamentally different mechanisms.

Comparison across different studies on native Korean readers indicates interesting patterns of results. Recently, Wang, Zhou, Shu, and Yan (2014) tested parafoveal processing for unbalanced Korean-Chinese bilinguals and found only low-level orthographic but not high-level semantic PB from Chinese preview words. In contrast, native Korean readers can obtain high-level (i.e., syntactic and semantic) information from parafoveally presented Korean words, as demonstrated by Kim et al. (2012) and in the present study. Taken together, it is likely that effectiveness in parafoveal high-level information processing is jointly influenced by factors at different levels, including a visual level, such as text layout; a linguistic level, such as priority in information processing in a writing system; and, finally, an individual level, such as reading proficiency. It will be of great interest for future research to test whether native Korean readers obtain semantic PB in reading Korean and to explore the similarity and specificity in parafoveal processing across different writing systems.

Author note This research was supported by Deutsche Forschungsgemeinschaft Grant (KL 955/18), by Natural Science Foundation of China $(31271082,81461130018)$, and by Natural Science Foundation of Beijing (7132119).

\section{References}

Altarriba, J., Kambe, G., Pollatsek, A., \& Rayner, K. (2001). Semantic codes are not used in integrating information across eye fixations in reading: Evidence from fluent Spanish-English bilinguals. Perception \& Psychophysics, 63, 875-890.

Altarriba, J., \& Mathis, K. M. (1997). Conceptual and lexical development in second language acquisition. Journal of Memory \& Language, 36, 550-568.

Bates, D., Maechler, M., Bolker, B., \& Walker, S. (2014). lme4: Linear mixed-effects models using Eigen and $S 4$ [R package version 1.1-7]. http://cran.r-project.org/web/packages/lme4/index.html

Chen, H.-C., \& Shu, H. (2001). Lexical activation during the recognition of Chinese characters: Evidence against early phonological activation. Psychonomic Bulletin \& Review, 8, 511-518.

Engbert, R., \& Kliegl, R. (2003). Microsaccades uncover the orientation of covert attention. Vision Research, 43, 1035-1045. doi:10.1016/ S0042-6989(03)00084-1

Hohenstein, S., \& Kliegl, R. (2014). Semantic preview benefit during reading. Journal of Experimental Psychology: Learning, Memory, and Cognition, 40, 166-190. doi:10.1037/a0033670

Hohenstein, S., Laubrock, J., \& Kliegl, R. (2010). Semantic preview benefit during eye movements in reading: A parafoveal fastpriming study. Journal of Experimental Psychology: Learning, Memory, and Cognition, 36, 1150-1170.

Hoosain, R. (1991). Psycholinguistic implications for linguistic relativity: A case study of Chinese. Hillsdale, NJ: Erlbaum.

Inhoff, A. W. (1982). Parafoveal word perception: A further case against semantic preprocessing. Journal of Experimental Psychology: Human Perception and Performance, 8, 137-145. 
Inhoff, A. W. (1984). Two stages of word processing during eye fixations in the reading of prose. Journal of Verbal Learning and Verbal Behavior, 23, 612-624.

Inhoff, A. W., \& Rayner, K. (1980). Parafoveal word perception: A case against semantic preprocessing. Perception \& Psychophysics, 27, 457-464.

Kim, Y. S., Radach, R., \& Vorstius, C. (2012). Eye movements and parafoveal processing during reading in Korean. Reading and Writing, 25, 1053-1078.

Kliegl, R., Masson, M. E. J., \& Richter, E. M. (2010). A linear mixed model analysis of masked repetition priming. Visual Cognition, 18, 655-681. doi:10.1080/13506280902986058

Libben, M., \& Titone, D. (2009). Bilingual language processing in context: Evidence from eye movement recordings during reading. Journal of Experimental Psychology: Learning, Memory, \& Cognition, 35, 381-390.

National Institute of Korean Language. (2005). Modern Korean usage frequency survey. Retrieved from http://www.korean.go.kr

R Development Core Team. (2014). R: A language and environment for statistical computing. Wien, Germany: R Foundation for Statistical Computing.

Rayner, K. (1975). The perceptual span and peripheral cues during reading. Cognitive Psychology, 7, 65-81. doi:10.1016/0010-0285(75) 90005-5

Rayner, K., Balota, D. A., \& Pollatsek, A. (1986). Against parafoveal semantic preprocessing during eye fixations in reading. Canadian Journal of Psychology, 40, 473-483.

Schotter, E. R. (2013). Synonyms provide semantic preview benefit in English. Journal of Memory and Language, 69, 619-633.

Schotter, E. R., Angele, B., \& Rayner, K. (2012). Parafoveal processing in reading. Attention, Perception, \& Psychophysics, 74, 5-35.

Sohn, H.-M. (2001). The Korean language. Cambridge, MA: Cambridge University Press.

Tsai, J.-L., Kliegl, R., \& Yan, M. (2012). Parafoveal semantic information extraction in traditional Chinese reading. Acta Psychologica, 141, 17-23. doi:10.1016/j.actpsy.2012.06.004
Wang, A., Zhou, W., Shu, H., \& Yan, M. (2014). Reading proficiency modulates parafoveal processing efficiency: Evidence from reading Chinese as a second language. Acta Psychologica, 152, 29-33. doi: 10.1016/j.actpsy.2014.07.010

Yan, M., Pan, J., Bélanger, N., \& Shu, H. (2015). Chinese deaf readers have early access to parafoveal semantics. Journal of Experimental Psychology: Learning, Memory, and Cognition, 41, 254-261. doi: 10.1037/xlm0000035

Yan, M., Richter, E. M., Shu, H., \& Kliegl, R. (2009). Chinese readers extract semantic information from parafoveal words during reading. Psychonomic Bulletin \& Review, 16, 561-566. doi:10.3758/PBR. 16.3.561

Yan, M., \& Sommer, W. (2015). Parafoveal-on-foveal effects of emotional word semantics in reading Chinese sentences: Evidence from eye movements. Journal of Experimental Psychology: Learning, Memory, and Cognition. doi:10.1037/xlm0000095

Yan, M., Zhou, W., Shu, H., \& Kliegl, R. (2012). Lexical and sub-lexical semantic preview benefits in Chinese reading. Journal of Experimental Psychology: Learning, Memory, and Cognition, 38, 1069-1075. doi:10.1037/a0026935

Yang, J., Wang, S., Tong, X., \& Rayner, K. (2012). Semantic and plausibility effects on preview benefit during eye fixations in Chinese reading. Reading and Writing, 25, 1031-1052. doi:10.1007/ s11145-010-9281-8

Yen, M.-H., Tsai, J.-L., Tzeng, O. J.-L., \& Hung, D. L. (2008). Eye movements and parafoveal word processing in reading Chinese sentences. Memory and Cognition, 36, 1033-1045. doi:10.3758/ MC.36.5.1033

Zhang, J. Y., Zhang, T., Xue, F., Liu, L., \& Yu, C. (2007). Legibility of Chinese characters and its implications for visual acuity measurement in Chinese reading population. Investigative Ophthalmology \& Visual Science, 48, 2383-2390.

Zhou, X., \& Marslen-Wilson, W. (2000). The relative time course of semantic and phonological activation in reading Chinese. Journal of Experimental Psychology: Learning, Memory, \& Cognition, 26, 1245-1265. doi:10.1037//0278-7393.26.5.1245 